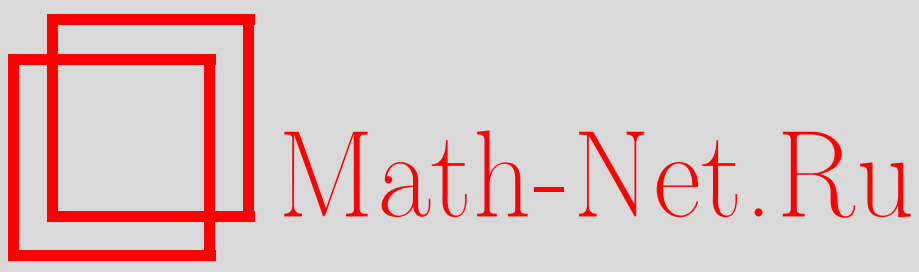

С. Е. Пастухова, Обоснование закона Дарси для пористой среды с условием неполного прилипания, Матем. сб., 1998, том 189, номер 12, 135-153

DOI: https://doi.org/10.4213/sm372

Использование Общероссийского математического портала Math-Net.Ru подразумевает, что вы прочитали и согласны с пользовательским соглашением

http://www . mathnet.ru/rus/agreement

Параметры загрузки:

IP: 35.174 .16 .151

26 апреля 2023 г., 14:59:23 
УДК 517.953

\author{
С.Е. Пастухова
}

\title{
Обоснование закона Дарси для пористой среды с условием неполного прилипания
}

\begin{abstract}
Изучена стационарная система Стокса в периодически перфорированной области с граничными условиями смешанного типа, которая описьвает движение вязкой несжимаемой жидкости в пористой среде, когда между жидкостью и стенками пор действует трение. Для этой задачи установлено соотношение между главньми членами из асимптотического разложения по $\varepsilon$ для скорости движения жидкости и для давления, где $\varepsilon$ - параметр, характеризующий степень измельченности пористой среды.

Библиографиия: 16 названий.
\end{abstract}

\section{$\S 1$. Введение}

Известно (см. [1]), что течение вязкой несжимаемой жидкости в пористых средах подчинено феноменологическому закону Дарси, связьвающему скорость движения жидкости с градиентом давления через тензор проницаемости. Информация о мелкомасштабной структуре пористой среды, особенности ее геометрии учитываются в коэффициентах этого тензора проницаемости, дающего усредненную характеристику сложно устроенной среды, в которой движется жидкость. Математическое обоснование возможности такого усредненного подхода, само определение усредненной характеристики среды - тензора проницаемости - приводят к изучению асимптотического поведения точного решения системы уравнений гидродинамики в областях со сложной структурой, называемых часто перфорированньми (см. [2]-[5]), где в случае периодически перфорированных областей в качестве малого параметра выступает размер периода перфорации. Обоснование закона Дарси при различных предположениях на структуру пористости среды можно найти, например, в [2; приложение Л. Тартара], [6], [7]. В перечисленных работах рассматривались решения системы Стокса в пористой среде с однородными условиями Дирихле для скорости движения жидкости на границе пор, при этом исследовалась асимптотика решений при гомотетическом измельчении пористой среды. Заметим, что в этой задаче однородное условие Дирихле для скорости движения на границе пор соответствует тому, что стенки пор непроницаемы для жидкости и жидкость к ним прилипает.

Отход от однородных условий Дирихле для скорости движения жидкости был сделан в [8], где краевое условие на границе пор вообще отсутствует. В [8] изучалась система Стокса с потенциалом, имеющим носитель в области, занятой твердой фракцией пористой среды. Наличие такого потенциала в уравнениях Стокса физически соответствует тому, что жидкость может проникать в твердую фракцию пористой среды. Тем не менее, твердая фракция пористой среды оказывает 
сопротивление течению жидкости, а введенньй в уравнения потенциал как раз и описывает это сопротивление. При этом классическому условию прилипания на границе соответствует потенциал, значения которого бесконечны на его носителе.

Есть другой случай, ког да приходится изучать уравнения Стокса без классического однородного условия Дирихле для скорости движения жидкости на границе пор. Рассмотрим пористую среду с непроницаемым твердым скелетом, когда нет полного прилипания жидкости к стенкам пор. А именно, будем считать, что касательное перемешение жидкости вдоль стенок пор возможно, но это перемещение тормозится силой трения, пропорциональной скорости движения жидкости (заметим, что в силу непроницаемости твердого скелета нормальная составляющая скорости движения на границе пор нулевая). Задачи такого сорта рассматривались, например, в гидродинамической теории смазки (см. [9; гл. II], а также [10]). Часто коэффициент пропорциональности для силы касательного трения оказывается реально большой величиной и его полагают тогда бесконечным. При таком переходе условие на касательное трение между жидкостью и твердой фракцией превращается в условие полного прилипания на гранище пор. Таким образом, эту задачу Стокса с условием неполного прилипания на стенках пор так же, как и разновидность задачи Стокса, изученную в [8], можно рассматривать как релаксацию классической задачи Стокса с условием полного прилипания на границе пор.

Хотя замена реальной ситуации, когда действует касательное трение между жидкостью и твердой фракцией пористой среды, при больших значениях коэффициента трения на более изученный случай, когда жидкость прилипает к стенкам пор, часто практикуется, все же, по-видимому, не стоит этого делать тогда, когда граница пористой среды составлена из разнородных (в смысле трения с жидкостью) материалов: внешняя граница области $\Omega$, в которой изучается движение жидкости, соответствует материалу со значительно большим коэффициентом трения, чем коэффициент трения на границе пор, заполняющих эту область $\Omega$. Такая замена существенно огрубляет усредненную картину движения жидкости, поскольку приводит к искажению тензора проницаемости. Оказывается, в указанных вьшше условиях неполного прилипания жидкости на границе пор усредненное описание ее течения в форме закона Дарси так же возможно, как и в классическом случае. При этом так же, как в классическом случае, тензор проницаемости пористой среды вбирает в себя информацию о мелкомасштабной структуре этой среды, но оказывается отличным от того тензора, который получается в классическом случае.

Краевая задача, описьвающая движение вязкой несжимаемой жидкости в пористой среде, заполняющей фиксированную область $\Omega$, при условии неполного прилипания жидкости на границе пор и полного прилипания жидкости на внешней границе $\partial \Omega$, будет точно поставлена в $\S 2$ (см. п. 2.4). Решением этой задачи является пара функций $\left(u^{\varepsilon}, p^{\varepsilon}\right)$, где $u^{\varepsilon}=\left(u_{1}^{\varepsilon}, \ldots, u_{d}^{\varepsilon}\right)$ - вектор-функция скорости движения жидкости, $p^{\varepsilon}$ - функция давления. Функции $u^{\varepsilon}, p^{\varepsilon}$ зависят от параметра $\varepsilon$, характеризующего периодическое измельчение пористой среды. Основной результат настоящей работы состоит в следуюшем. Во-первых, при $\varepsilon \rightarrow 0$ функции $u^{\varepsilon}, p^{\varepsilon}$ имеют тот же порядок (второй и нулевой, соответственно) малости по $\varepsilon$, что и в случае однородного условия Дирихле на всей границе области, в которой рассматривается решение задачи Стокса. Таким образом, условие непроницае- 
мости стенок пор, общее для обоих случаев (полного и неполного прилипания), в которых рассматривается задача Стокса, является решающим при определении порядка малости функций $u^{\varepsilon}, p^{\varepsilon}$. Во-вторых, при $\varepsilon \rightarrow 0$ пара функций $\left(\varepsilon^{-2} u^{\varepsilon}, p^{\varepsilon}\right)$ сходится в определенном смыслек $(u, p)$, где $(u, p)$-пара функций $\left(u=\left(u_{1}, \ldots, u_{d}\right)\right.$ - вектор-функция), которая находится как решение следуюшей задачи:

$$
\begin{gathered}
\operatorname{div} u=0 \text { в } \Omega, \\
u_{i}=\sum_{j=1}^{d} K_{i j}\left(f_{j}-\frac{\partial p}{\partial x_{j}}\right) \text { в } \Omega, \\
u_{n}=u \cdot n=0 \text { на } \partial \Omega,
\end{gathered}
$$

где $n$ - вектор внешней единичной нормали к $\partial \Omega, f=\left(f_{1}, \ldots, f_{d}\right)$ - заданный вектор внешних сил, действующих на жидкость. Тензор $K$ с постоянными компонентами $K_{i j}, i, j=1, \ldots, d$, называемый тензором проницаемости пористой средь, не зависит от $\varepsilon$, а также от функции $f$ и области $\Omega$. Тензор $K$ зависит от геометрии периодической структуры пор. При этом выясняется любопытный факт, что тензор $K$ не зависит от коэффициента касательного трения $\lambda$ между жидкостью и твердой фракцией, если считать, что величина $\lambda$ остается неизменной при измельчении пор, когда параметр $\varepsilon$ стремится к нулю. (Если отказаться от условия неизменности $\lambda$ при $\varepsilon \rightarrow 0$ и считать $\lambda$ вторым изменяющимся параметром задачи Стокса, то при определенных соотношениях между $\lambda$ и $\varepsilon$ тензор $K$ будет зависеть от $\lambda$. Это имеет место тогда, когда $\lambda=\lambda(\varepsilon)=\lambda_{0} \varepsilon^{-1}, \lambda_{0}=$ const при $\varepsilon \rightarrow 0$. Но случай, когда $\lambda$ - изменяюшийся параметр в задаче Стокса, остается за рамками настояшей работы.) Таким образом, главное приближение к функции $u^{\varepsilon}$ как бы не замечает существуюшего трения между жидкостью и твердой фракцией. На макроуровне (который в данном случае порядка $\varepsilon^{2}$ ) трение как будто отсутствует вовсе, каким бы коэффициентом $\lambda$ это трение ни характеризовалось, лишш бы этот коэффициент $\lambda$ был бы неизменным при $\varepsilon \rightarrow 0$.

Заметим, что если рассматривать в асимптотическом разложении функции $u^{\varepsilon}$ по степеням $\varepsilon$ следуюшие члены вслед за главным, то они уже будут зависеть от коэффициента трения $\lambda$.

Предельные соотношения (1)-(3) для изучаемой задачи Стокса имеют такой же вид, что и для аналогичной задачи Стокса с условиями прилипания на всей границе области, отличие лишш в тензоре $K$. Но тензор $K$ оказывается и в этой задаче положительно определенным и симметричным. Поэтому задача (1)-(3) относительно функции предельного давления $p$ является классической задачей для эллиптического дифференциального оператора второго порядка в области $\Omega$ с краевым условием Неймана на границе $\partial \Omega$. Вектор-функция $u$ первого приближения к скорости движения жидкости определяется через предельную функцию давления $p$ по формуле (2). Эта формула и выражает суть закона Дарси движения вязкой несжимаемой жидкости в пористой среде в сформулированных выше предположениях.

Скажем коротко о структуре настоящей работы. Следуя традициям [11], [12] и более ранних работ Лере (см. библиографию в [11], [12]), естественно изучать 
задачу Стокса в обобшенной (вариационной) формулировке (см. п. 2.4). Для этого прежде всего необходимо ввести адекватные для задачи функциональные пространства, и это сделано в пा. 2.1-2.4. В $\S 3$ доказана теорема существования и единственности изучаемой задачи, а также утверждение о равномерной ограниченности по параметру $\varepsilon$ в определенных нормах пары $\left(\varepsilon^{-2} u^{\varepsilon}, p^{\varepsilon}\right)$, составленной из решений изучаемой задачи. В $\S 4$ дано обоснование закона Дарси для пористой среды в сформулированных выше предположениях. Для этого прежде всего изучается предельная задача (1)-(3), ее свойства. Далее показывается, что задача (1)-(3) - усредненная для исходной задачи Стокса в пористой среде в смысле сходимости при $\varepsilon \rightarrow 0$ функций $\varepsilon^{-2} u^{\varepsilon}$ и $p^{\varepsilon}$ к решениям $u$ и $p$ задачи (1)-(3). Для доказательства этого факта используется модифицированный метод Тартара (см. приложение Л. Тартара в [2]). При этом так же, как в [2], существенно используется построенный Тартаром оператор продолжения функции давления $p$ с области $\Omega_{\varepsilon}$ на всю область $\Omega$. Этот момент в доказательстве основных результатов предполагает более ограничительные требования на структуру перфорированной области $\Omega_{\varepsilon}$, чем те, в которых изучалась в стационарном случае задача Стокса в [6] и нестационарная задача Стокса в [13]. В [6] и затем в [13] сходимость решений задачи Стокса в области $\Omega_{\varepsilon}$ к решениям краевой задачи, поставленной во всей области $\Omega$, выводилась из оценки погрешности второго приближения к точному решению. А эта оценка основывалась на равномерной по $\varepsilon$ оценке между нормами функций в пространствах $H^{-1}\left(\Omega_{\varepsilon}\right)$ и $L^{2}\left(\Omega_{\varepsilon}\right) / \mathbb{R}$, доказанной в [6]. Представляется возможньм осушествить этот подход и в задаче Стокса с условием неполного прилипания и получить за счет этого перечисленные выше результаты в перфорированных областях более общей геометрии. В настоящей же работе автору хотелось продемонстрировать при изучении поставленной задачи Стокса возможности метода Тартара компенсированной компактности, которьй и ранее успешно применялся при исследовании различных вопросов теории усреднения.

\section{§2. Обобщенная постановка задачи Стокса с условием неполного прилипания и связанные с этой задачей функциональные пространства}

2.1. Необходимые функциональные пространства и их свойства. В дальнейшем будем использовать стандартные обозначения, а также обозначения из [12].

Пусть $Q$ - ограниченная область в $\mathbb{R}^{d}$ с липшицевой границей $\partial Q, D_{i}=\partial / \partial x_{i}$, $i=1, \ldots, d$.

Введем пространство $\mathscr{V}(Q)=\left\{u \in D(Q)^{d}: \operatorname{div} u=0\right\}$, тогда $H(Q), V(Q)$ есть замыкания пространства $\mathscr{V}(Q)$ по норме $L^{2}(Q)^{d}, H^{1}(Q)^{d}$, соответственно. Введем также, следуя [12], вспомогательное пространство $E(Q)=\left\{u \in L^{2}(Q)^{d}\right.$ : $\left.\operatorname{div} u \in L^{2}(Q)\right\}$.

Пусть далее $n=\left(n_{1}, \ldots, n_{d}\right)$ - вектор внешшей единичной нормали к границе $\partial Q$. Тогда (см. в [12; гл. I, $\S 1$, п. 1.3]) определен оператор следа $\gamma_{n} \in \mathscr{L}\left(E(Q), H^{-1 / 2}(\partial Q)\right)$ такой, что $\gamma_{n} u=\left.u \cdot n\right|_{\partial Q}$ для любой функции $u \in C^{\infty}(\bar{Q})^{d}$. Здесь и всюду далее для векторов $v, w \in \mathbb{R}^{d}$ обозначаем через $v \cdot w$ их скалярное произведение. 
Оказывается, что (см. в $\left[12 ;\right.$ гл. I, $\S 1$, п. 1.4]) $H(Q)=\left\{u \in L^{2}(Q)^{d}: \operatorname{div} u=0\right.$, $\left.\gamma_{n} u=0\right\}, V(Q)=\left\{u \in H_{0}^{1}(Q)^{d}: \operatorname{div} u=0\right\}$. Кроме того, справедливы следующие утверждения [12; гл. I, $\S 1$, предложения 1.1, 1.2].

ПРЕДЛОЖЕНИЕ 1. Пусть $f \in D^{\prime}(Q)^{d}$. Тогда для того итобъц $f=\operatorname{grad} p \partial \Omega я$ некоторой функции $p \in D^{\prime}(Q)$, необходимо и достаточно, чтобъц $\langle f, v\rangle=0$ для любой функции $v \in \mathscr{V}(Q)$ в смисле распределений.

ПРЕДЛОЖЕНИЕ 2. Пусть $p \in D^{\prime}(Q), D_{i} p \in H^{-1}(Q), i=1, \ldots, d$. Тогдa $p \in L^{2}(Q)$ и верна оченка

$$
|p|_{L^{2}(Q) / \mathbb{R}} \leqslant C|\operatorname{grad} p|_{H^{-1}(Q)^{d}},
$$

əде $C=\operatorname{const}(Q)$, а факторпространство

$$
L^{2}(Q) / \mathbb{R}=\left\{p \in L^{2}(Q): \int_{Q} p d x=0\right\}
$$

рассматривается с естественной $L^{2}$-нормой.

2.2. Вариант формулы Грина-Стокса. Определим для вектор-функции $u(x)=\left(u_{1}(x), \ldots, u_{d}(x)\right)$ и скалярной функции $p(x)$ тензоры $e_{i k}(u)=D_{i} u_{k}+D_{k} u_{i}$,

$$
T_{i k}(u, p)=-\delta_{i k} p(x)+\nu e_{i k}(u),
$$

где $\nu$ - некоторая положительная константа (именуемая далее кинематическим коэффициентом вязкости), связанная с изучаемой задачей течения жидкости, $\delta_{i k}$ - символ Кронекера, т.е. $\delta_{i k}=0$, если $i \neq k, \delta_{i k}=1$, если $i=k$. Принято назьвать тензор $T_{i k}(u, p)$ тензором напряжения, соответствующим течению жидкости со скоростью $u(x)$ и давлением $p(x)$.

Для гладких в $\bar{Q}$ и соленоидальных полей $u(x), \varphi$ и гладкой функции $p(x)$ нетрудно проверить, что (см. [11; с. 72-73])

$$
D_{k}\left(T_{i k}(u, p) \varphi_{i}\right)=\frac{\nu}{2} e_{i k}(u) e_{i k}(\varphi)+\left(\nu \triangle u_{i}-D_{i} p\right) \varphi_{i}
$$

где $\triangle$ - оператор Лапласа, а по повторяюшимся индексам здесь и далее подразумевается суммирование от 1 до $d$. После интегрирования (5) по области $Q$ получаем интегральное равенство, которое есть вариант формулы Грина-Стокса:

$$
\int_{Q}\left(\nu \triangle u_{i}-D_{i} p\right) d x=-\frac{\nu}{2} \int_{Q} e_{i k}(u) e_{i k}(\varphi) d x+\int_{\partial Q} T_{i k}(u, p) \varphi_{i} n_{k} d s
$$

2.3. Перфорированные области $\Omega_{\varepsilon}$. Далее в качестве области $Q$ будет фигурировать перфорированная область $\Omega_{\varepsilon}$, зависяшая от малого положительного параметра $\varepsilon$. Область $\Omega_{\varepsilon}$ моделирует пористую среду. Считаем, что $\Omega_{\varepsilon}-$ перфорированная область I типа по классификации [4] (см. [4; с. 38-39]). Напомним, что это означает.

Пусть $\Omega$ - гладкая ограниченная область в $\mathbb{R}^{d}$ и $\omega$-гладкая неограниченная область в $\mathbb{R}^{d}$ с 1-периодической структурой, $B=\left\{x \in \mathbb{R}^{d}:\left|x_{i}\right|<\frac{1}{2}, i=1, \ldots, d\right\}$ - единичный куб в $\mathbb{R}^{d}, P=\omega \cap B$. Будем предполагать, что граница $S=\partial(B \backslash P)$ отстоит от границы $\partial B$ на положительном расстоянии. Если $\varepsilon \omega=\left\{x \in \mathbb{R}^{d}\right.$ : $\left.\varepsilon^{-1} x \in \omega\right\}$, то пусть $\Omega_{\varepsilon}=\Omega \cap \varepsilon \omega$. Граница $\partial \Omega_{\varepsilon}=\Gamma_{\varepsilon} \cup S_{\varepsilon}$, где $\Gamma_{\varepsilon}=\partial \Omega \cap \varepsilon \omega$, $S_{\varepsilon}=\partial(\varepsilon \omega) \cap \Omega$. 


\section{4. Обобщенная постановка задачи Стокса с условием неполного} прилипания на границе. Если в качестве области $Q$ брать перфорированные области $\Omega_{\varepsilon}$ при различных значениях параметра $\varepsilon, \varepsilon \in(0,1), \varepsilon \rightarrow 0$, то мы получаем серию пространств, введенных в п. 2.1, которые определены на перфорированных областях $\Omega_{\varepsilon}$. В дополнение к этой серии пространств, зависящих от параметра $\varepsilon$ через область $\Omega_{\varepsilon}$, введем пространство $W_{\varepsilon}=W\left(\Omega_{\varepsilon}\right)$, тесно связанное с изучаемой задачей Стокса в области $\Omega_{\varepsilon}$. Пространство $W_{\varepsilon}$ будет промежуточным между $H\left(\Omega_{\varepsilon}\right)$ и $V\left(\Omega_{\varepsilon}\right)$, если

$$
W_{\varepsilon}=W\left(\Omega_{\varepsilon}\right)=H\left(\Omega_{\varepsilon}\right) \cap H^{1}\left(\Omega_{\varepsilon}, \Gamma_{\varepsilon}\right)^{d}
$$

где $H^{1}\left(\Omega_{\varepsilon}, \Gamma_{\varepsilon}\right)^{d}$ есть замькание по норме $H^{1}\left(\Omega_{\varepsilon}\right)^{d}$ пространства $C^{\infty}\left(\Omega_{\varepsilon}, \Gamma_{\varepsilon}\right)^{d}$ гладких вектор-функций, равных нулю в некоторой окрестности $\Gamma_{\varepsilon}$. Тогда, используя свойство пространства $H\left(\Omega_{\varepsilon}\right)$, получаем $W_{\varepsilon}=\left\{u \in H^{1}\left(\Omega_{\varepsilon}, \Gamma_{\varepsilon}\right)^{d}\right.$ : $\left.\operatorname{div} u=0,\left.\gamma_{n} u\right|_{S_{\varepsilon}}=0\right\}$. Первоначально можно считать, что норма в $W_{\varepsilon}-$ это стандартная норма, унаследованная из $H^{1}\left(\Omega_{\varepsilon}\right)^{d}$.

Рассмотрим последовательность задач, зависящих от параметра $\varepsilon$. Это будут задачи Стокса с условием неполного прилипания в области $\Omega_{\varepsilon}$ относительно неизвестных функций $u^{\varepsilon}, p^{\varepsilon}$ :

$$
\begin{gathered}
-\nu \Delta u^{\varepsilon}+\operatorname{grad} p^{\varepsilon}=f \text { в } \Omega_{\varepsilon}, \\
\operatorname{div} u^{\varepsilon}=0 \text { в } \Omega_{\varepsilon}, \\
u^{\varepsilon}=0 \text { на } \Gamma_{\varepsilon}, \\
u^{\varepsilon} \cdot n=0, \quad T_{\tau}\left(u^{\varepsilon}, p^{\varepsilon}\right)+\lambda u^{\varepsilon}=0 \text { на } S_{\varepsilon} .
\end{gathered}
$$

Здесь вектор-функция $u^{\varepsilon}-$ скорость движения жидкости, $p^{\varepsilon}-$ давление в жидкости, $\nu$ - кинематический коэффициент вязкости, $f$ - заданный вектор внешних сил, $T_{\tau}\left(u^{\varepsilon}, p^{\varepsilon}\right)$ - касательная компонента вектора напряжений на $S_{\varepsilon}$. Определим вектор $T_{\tau}\left(u^{\varepsilon}, p^{\varepsilon}\right)$ точно. При движении вязкой несжимаемой жидкости со скоростью $u^{\varepsilon}$ и давлением $p^{\varepsilon}$ на поверхности $S_{\varepsilon}$ действует напряжение $T=T\left(u^{\varepsilon}, p^{\varepsilon}\right)$. Это вектор-функция, компоненты которой

$$
T_{i}=T_{i}\left(u^{\varepsilon}, p^{\varepsilon}\right)=T_{i k}\left(u^{\varepsilon}, p^{\varepsilon}\right) n_{k}, \quad i=1, \ldots, d
$$

определяются через вектор $n$ единичной внешней нормали к $S_{\varepsilon}$ и компоненты тензора напряжений $T_{i k}\left(u^{\varepsilon}, p^{\varepsilon}\right)$, введенные в формуле (4). Вектор напряжений $T$, как всякий вектор, может быть разложен на две взаимно перпендикулярные составляющие:

$$
T=T_{n} n+T_{\tau}
$$

Здесь $T_{n}=T \cdot n$ - проекция вектора $T$ на внешнюю единичную нормаль $n, T_{\tau}$ - касательная к $S_{\varepsilon}$ составляющая вектора $T$. Эта касательная составляющая $T_{\tau}=T_{\tau}\left(u^{\varepsilon}, p^{\varepsilon}\right)$ как раз и присутствует в краевом условии (10). Коэффициент $\lambda$ в краевом условии (10) - положительная константа - коэффициент касательного трения между жидкостью и стенками пор $S_{\varepsilon}$. 
Граничное условие (9) соответствует полному прилипанию жидкости на границе $\Gamma_{\varepsilon}$, а граничное условие (10) означает, что жидкость не проникает через границу $S_{\varepsilon}$, но и не прилипает к ней, а ее возможное в касательном направлении к $S_{\varepsilon}$ движение подвержено силе трения с коэффициентом $\lambda$. Заметим, что поскольку $u^{\varepsilon} \cdot n=0$ на $S_{\varepsilon}$, то $\left.u^{\varepsilon}\right|_{S_{\varepsilon}}$ лежит в касательной плоскости к $S_{\varepsilon}$ и разложение для вектора $\left.u^{\varepsilon}\right|_{S_{\varepsilon}}$, аналогичное (11), тривиально, т.е. в нем отсутствует первое слагаемое - нормальная к $S_{\varepsilon}$ компонента.

В дальнейшем полезно иметь в виду представление для скалярного произведения вектора напряжений $T$ и какого-то другого вектора $\varphi$ в следующем виде:

$$
T \cdot \varphi=T_{\tau} \cdot \varphi_{\tau}+T_{n} \varphi_{n}
$$

которое вьводится, если использовать разложение (11) и аналогичное ему разложение для вектора $\varphi: \varphi=\varphi_{n} n+\varphi_{\tau}$.

Установим, какую обобщенную постановку задачи (7)-(10) естественно ввести. Для этого, считая $u^{\varepsilon}, p^{\varepsilon}, f$ гладкими функциями, удовлетворяюшими $(7)-(10)$, умножим (7) скалярно на произвольную соленоидальную функцию $\varphi \in C^{\infty}\left(\Omega_{\varepsilon}, \Gamma_{\varepsilon}\right)^{d}$. Тогда получим, используя формулу (6), граничные условия (9) и (10), а также определение вектора напряжений $T$, его разложение (11) и формулу (12), следующее интегральное равенство:

$$
\int_{\Omega_{\varepsilon}} f \cdot \varphi d x=\frac{\nu}{2} \int_{\Omega_{\varepsilon}} e_{i k}\left(u^{\varepsilon}\right) e_{i k}(\varphi) d x+\lambda \int_{S_{\varepsilon}} u^{\varepsilon} \cdot \varphi d s .
$$

Заметим, что второе слагаемое в правой части (13) можно заменить на $\lambda \int_{S_{\varepsilon}} u_{\tau} \cdot \varphi_{\tau} d s$, так как $u_{\varepsilon} \cdot n=\left(u_{\varepsilon}\right)_{n}=0$ и верна формула (12).

Далее для любых функций $\psi, \varphi \in L^{2}\left(\Omega_{\varepsilon}\right)^{d}$ положим

$$
(\psi, \varphi)_{\varepsilon}=\int_{\Omega_{\varepsilon}} \psi \cdot \varphi d x
$$

а для любых функций $\psi, \varphi \in H^{1}\left(\Omega_{\varepsilon}\right)^{d}$ положим

$$
[\psi, \varphi]_{\varepsilon}=\frac{\nu}{2} \int_{\Omega_{\varepsilon}} e_{i k}(\psi) e_{i k}(\varphi) d x+\lambda \int_{S_{\varepsilon}} \psi \cdot \varphi d s .
$$

Тогда интегральное равенство (13) можно переписать в компактном виде:

$$
(f, \varphi)_{\varepsilon}=\left[u^{\varepsilon}, \varphi\right]_{\varepsilon} .
$$

Вьвод интегрального равенства (14) для случая гладких функций $u^{\varepsilon}, p^{\varepsilon}, f$ показывает, что естественно ввести следуюшее понятие обобшенного решения задачи (7) $-(10)$.

ОПРЕДЕЛЕНИЕ 1. Функция $u^{\varepsilon} \in W_{\varepsilon}$ есть (обобщенное) решение задачи (7)-(10), если для любой функции $\varphi \in W_{\varepsilon}$ выполнено равенство (14). 
ПРЕДЛОЖЕНИЕ 3. Сформулированные ниже условия (I), (II) әквивалентHbl:

(I) $u^{\varepsilon} \in W_{\varepsilon}$ и удовлетворяет (14) для любой функиии $\varphi \in W_{\varepsilon}$;

(II) $u^{\varepsilon} \in H^{1}\left(\Omega_{\varepsilon}, \Gamma_{\varepsilon}\right)^{d}$ и удовлетворяет (7)-(10) в следующем смисле: существует функция $p^{\varepsilon} \in L^{2}\left(\Omega_{\varepsilon}\right) / \mathbb{R}$ такая, что

$$
\begin{gathered}
-\nu \triangle u^{\varepsilon}+\operatorname{grad} p^{\varepsilon}=f \text { в } \Omega_{\varepsilon} \text { в смысле теории распределений, } \\
\operatorname{div} u^{\varepsilon}=0 \text { в } \Omega_{\varepsilon} \text { в смисле теории распределений, } \\
\gamma_{n} u^{\varepsilon}=0 \text { на } S_{\varepsilon}, T_{\tau}\left(u^{\varepsilon}, p^{\varepsilon}\right)+\lambda u^{\varepsilon}=0 \text { на } S_{\varepsilon} \\
\text { в смисле интегрального равенства }(18) \text { (см. ниже }) .
\end{gathered}
$$

ДоКАЗАТЕЛЬСТво. Для вывода условия (I) из условия (II) нужно использовать те же рассуждения, что предваряют определение 1.

Докажем обратное: (I) $\Rightarrow$ (II). В самом деле, если выполнено условие (I), то, рассматривая в (14) $\varphi \in \mathscr{V}\left(\Omega_{\varepsilon}\right)$, выводим прежде всего уравнение (15), которому удовлетворяет функция $u^{\varepsilon}$ в паре с некоторой функцией $p^{\varepsilon} \in L^{2}\left(\Omega_{\varepsilon}\right) / \mathbb{R}$. Для этого необходимо сослаться на предложения 1 и 2 из п. 2.1. Выполнение условия (16) и первого условия из (17) очевидны, если $u^{\varepsilon} \in W_{\varepsilon}$. Используя полученные уже свойства функции $u^{\varepsilon} \in W_{\varepsilon}$, удовлетворяюшей равенству (14), получаем в силу формул (6), (11)

$$
\int_{S_{\varepsilon}}\left(T_{\tau}\left(u^{\varepsilon}, p^{\varepsilon}\right)+\lambda u^{\varepsilon}\right) \cdot \psi d s=0
$$

для любой функции $\psi \in H^{1 / 2}\left(S_{\varepsilon}\right)^{d-1}$. Здесь использован следующий факт: если $\gamma_{0} \in \mathscr{L}\left(H^{1}\left(\Omega_{\varepsilon}\right)^{d}, H^{1 / 2}\left(\partial \Omega_{\varepsilon}\right)^{d}\right)$ - обычный оператор следа, который для вектор-функции $u \in C^{2}\left(\overline{\Omega_{\varepsilon}}\right)^{d}$ сопоставляет каждой ее компоненте $u_{i}$ обычное сужение $\left.u_{i}\right|_{\partial \Omega_{\varepsilon}}$ (см. [12; гл. I, $\S 1$, п. 1.3]), то в случае, когда функция $\varphi$ пробегает все пространство $W_{\varepsilon}$, значения оператора следа $\gamma_{0}$ на поверхности $\left.S_{\varepsilon} \gamma_{0}\right|_{S_{\varepsilon}}=\psi$ пробегают все пространство $H^{1 / 2}\left(S_{\varepsilon}\right)^{d-1}$. Таким образом, выполнение условия (17) показано и доказательство предложения 3 вместе с тем завершено.

\section{§3. Теорема существования и единственности}

\section{задачи Стокса (7)-(10). Равномерные оценки для ее решения}

3.1. Соотношения между различными нормами функций из $W_{\varepsilon}$. Так как пространство $W_{\varepsilon}$ есть подпространство $H^{1}\left(\Omega_{\varepsilon}\right)^{d}$, то для $u \in W_{\varepsilon}$ определена естественная норма

$$
|u|_{W_{\varepsilon}}=\left(\int_{\Omega_{\varepsilon}} u_{i} u_{i} d x+\int_{\Omega_{\varepsilon}} D_{i} u_{j} D_{i} u_{j} d x\right)^{1 / 2} .
$$

Но для изучения задачи $(7)-(10)$ удобно ввести в $W_{\varepsilon}$ другую норму

$$
\langle u\rangle_{\varepsilon}=\left(\int_{\Omega_{\varepsilon}} e_{i j}(u) e_{i j}(u) d x\right)^{1 / 2} .
$$

То, что это норма, будет видно из дальнейшего (см. неравенства (21) и (19)). Далее для $u \in L^{2}\left(\Omega_{\varepsilon}\right)^{d}$ используем обозначение: $|u|_{\varepsilon}=\left(\int_{\Omega_{\varepsilon}} u_{i} u_{i} d x\right)^{1 / 2}$. 
Лемма 1. При $\varepsilon \leqslant \varepsilon_{0}\left(\varepsilon_{0}-\right.$ константа, определяемая геометрией $\left.\Omega, \omega\right)$ для любой функции $v \in W_{\varepsilon}$ выполнено неравенство

$$
|v|_{\varepsilon} \leqslant C_{1} \varepsilon\langle v\rangle_{\varepsilon},
$$

где константа $C_{1}$ не зависит от $\varepsilon$ и от функции $v$.

Эта лемма вытекает из леммы 1 работы [14], где было установлено, что если $v \in H^{1}\left(\Omega_{\varepsilon}, \Gamma_{\varepsilon}\right)^{d}$, то для достаточно малых $\varepsilon \leqslant \varepsilon_{0}=\operatorname{const}(\Omega, \omega)$ имеет место оценка

$$
|v|_{\varepsilon}^{2} \leqslant C_{2}\left(\varepsilon \int_{S_{\varepsilon}} v_{n}^{2} d s+\varepsilon^{2}\langle v\rangle_{\varepsilon}^{2}\right)
$$

где $\left.v_{n}\right|_{S_{\varepsilon}}=\left.(v \cdot n)\right|_{S_{\varepsilon}}$ и константа $C_{2}$ не зависит от $\varepsilon$ и от функции $v$. В самом деле, неравенство (19) - очевидное следствие из неравенства (20), если в последнем $\left.v_{n}\right|_{S_{\varepsilon}}=0$.

Чтобы показать эквивалентность в пространстве $W_{\varepsilon}$ нормы $\langle\cdot\rangle_{\varepsilon}$ и стандартной нормы $|\cdot|_{W_{\varepsilon}}$, унаследованной из пространства $H^{1}\left(\Omega_{\varepsilon}\right)^{d}$, воспользуемся неравенством Корна (см. [4], а также [15]). Действительно, для перфорированных областей $\Omega_{\varepsilon}$, определенных в п. 2.3 , в [4] доказаны равномерные по $\varepsilon$ при достаточно малых $\varepsilon, \varepsilon \leqslant \varepsilon_{0}$, неравенства Корна

$$
\int_{\Omega_{\varepsilon}} e_{i j}(v) e_{i j}(v) d x+\int_{\Omega_{\varepsilon}} v_{i} v_{i} d x \geqslant C|v|_{H^{1}\left(\Omega_{\varepsilon}\right)^{d}}^{2}
$$

для любых $v \in H^{1}\left(\Omega_{\varepsilon}\right)^{d}$, где константа $C$ не зависит от $\varepsilon$. Если согласно (19) оценить левую часть (21) сверху через $2\langle v\rangle_{\varepsilon}^{2}$ при достаточно малых $\varepsilon$, то нужная для получения эквивалентности норм оценка оказывается очевидной.

\section{2. Теорема о существовании, единственности и равномерных оцен-} ках.

Tеорема 1. Пусть $f \in L^{2}\left(\Omega_{\varepsilon}\right)^{d}$, тогда для любъх $\varepsilon \leqslant \varepsilon_{0}$ существует $u$ единственно решение задачи (7)-(10) в смьсле определения 1. При этом для ее решения $u=u^{\varepsilon}$ выполнены оченки

$$
\begin{aligned}
& \langle u\rangle_{\varepsilon} \leqslant C_{3} \varepsilon|f|_{\varepsilon}, \\
& |u|_{\varepsilon} \leqslant C_{4} \varepsilon^{2}|f|_{\varepsilon},
\end{aligned}
$$

где константы $C_{3}, C_{4}$ не зависят от $\varepsilon$ и от функции $f$.

ДокАЗАТЕЛЬСТво. Воспользуемся интегральньм тождеством (14), которому удовлетворяет обобщенное решение задачи (7)-(10). Тогда правая часть его задает квадратичную форму на пространстве $W_{\varepsilon}$, а левая часть его при фиксированной функции $f$ задает линейный функционал на $W_{\varepsilon}$. Нетрудно проверить, что для этих квадратичной формы и функционала выполнены все условия теоремы Лакса-Мильграма (нужно воспользоваться оценкой (19) и рассмотреть $W_{\varepsilon}$ как пространство с нормой $\left.\langle\cdot\rangle_{\varepsilon}\right)$. Из теоремы Лакса-Мильграма вытекает существование и единственность обобщенного решения задачи (7)-(10), а также выполнение оценки (22). Из оценки (22) в силу (19) следует справедливость и оценки (23). 
СЛЕДСТВИЕ ТЕОРЕМЫ 1. Функция $p^{\varepsilon} \in L^{2}\left(\Omega_{\varepsilon}\right) / \mathbb{R}$ из условия (II) предложения 3 (обобщенная функция давления из задачи Стокса) удовлетворяет следующей оченке:

$$
\left|\nabla p^{\varepsilon}\right|_{H^{-1}\left(\Omega_{\varepsilon}\right)^{d}} \leqslant C_{5} \varepsilon|f|_{L^{2}\left(\Omega_{\varepsilon}\right)^{d}},
$$

где константа $C_{5}$ не зависит от $\varepsilon$ и от функции $f$ при достаточно мальх $\varepsilon \leqslant \varepsilon_{0}$.

ДоказАТЕЛЬство. Воспользуемся тем, что $\nabla p^{\varepsilon}=\nu \Delta u^{\varepsilon}+f$ в $\Omega_{\varepsilon}$ в смысле обобшенных функций, где $u^{\varepsilon}$ есть обобшенное решение задачи (7)-(10), для которого получены равномерные по $\varepsilon$ оценки $(22),(23)$. Поэтому в силу неравенства Корна $(21)$ и неравенства (19) из определения нормы в пространстве $H^{-1}\left(\Omega_{\varepsilon}\right)$ можно вьвести следующее:

$$
\left|\nabla p^{\varepsilon}\right|_{H^{-1}\left(\Omega_{\varepsilon}\right)} \leqslant C_{6}\left(\sum_{i=1}^{d}\left|D_{i} u^{\varepsilon}\right|_{L^{2}\left(\Omega_{\varepsilon}\right)^{d}}+\varepsilon|f|_{L^{2}\left(\Omega_{\varepsilon}\right)^{d}}\right) \leqslant \varepsilon C_{7}|f|_{L^{2}\left(\Omega_{\varepsilon}\right)^{d}}
$$

\section{§4. Закон Дарси для задачи Стокса с условием неполного прилипания}

4.1. Вспомогательная задача на ячейке периодичности и определение тензора проницаемости пористой среды. Введем на ячейке периодичности $P$ необходимые пространства. Пусть $\mathscr{V}_{\text {per }}(P)$ - пространство функций из $C^{\infty}(P)^{d}, 1$-периодических по всем переменньм и равных 0 в некоторой окрестности $S$, и пусть $H_{\mathrm{div}}^{1}(P)^{d}=\left\{u(y) \in H^{1}(P)^{d}: \operatorname{div} u=0, u(y)-1\right.$-периодические по всем переменным $\}$. Пусть также $H(P)$ есть замыкание $\mathscr{V}_{\text {per }}(P)$ по норме $L^{2}(P)^{d}$ и $V_{1}=H(P) \cap H^{1}(P)^{d}$. Как указывалось в п. 2.1, из [12] следует, что $V_{1}=\left\{u \in H_{\mathrm{div}}^{1}(P)^{d}: \gamma_{n} u=0\right.$ на $\left.S\right\}$, где $\gamma_{n}$ - оператор следа на границу $S$ и через $n$ в данном случае обозначен вектор единичной внешшей (по отношению к $P$ ) нормали к $S$, так что для $u \in C^{2}(\bar{P})^{d} \gamma_{n} u=\left.u \cdot n\right|_{S}$.

Рассмотрим следуюшие краевые задачи на $P$ при $i=1, \ldots, d$ :

$$
\begin{gathered}
-\nabla_{y} q^{i}+\Delta_{y} z^{i}+e^{i}=0 \text { в } P, \\
\operatorname{div}_{y} z^{i}=0 \text { в } P, \\
z^{i} \cdot n=0, \quad T_{\tau y}\left(z^{i}, q^{i}\right)=0 \text { на } S, \\
z^{i}(y)-1 \text {-периодична по } y .
\end{gathered}
$$

Здесь $e^{i}-i$-й единичный базисньй вектор прямоугольной системы координат, введенной в $\mathbb{R}^{d}, T_{\tau y}\left(z^{i}, q^{i}\right)$ - касательная составляющая вектора напряжения $T_{y}\left(z^{i}, q^{i}\right)$, а вектор напряжения $T_{y}\left(z^{i}, q^{i}\right)$ определяется по функциям $z^{i}$ и $q^{i}$ аналогично тому, как вектор $T(u, p)$ определялся по функциям $u$ и $p$ в п. 2.4, с той лишь разницей, что дифференцирование нужно вести по переменным $y$ и в качестве $n$ рассматривать вектор единичной внешней нормали к $S$. 
Пусть

$$
\begin{gathered}
{[v, w]_{P}=\frac{\nu}{2} \int_{P} e_{i k, y}(v) e_{i k, y}(w) d y} \\
e_{i k, y}(v)=\frac{\partial}{\partial y_{i}} v_{k}+\frac{\partial}{\partial y_{k}} v_{i}, \quad i, k=1, \ldots, d .
\end{gathered}
$$

Определим обобщенное решение задачи $\left(25_{i}\right), i=1, \ldots, d$.

ОПРЕДЕЛЕНИЕ 2. Функция $v \in V_{1}$ - обобщенное решение задачи $\left(25_{i}\right), i=$ $1, \ldots, d$, если для любой функции $w \in V_{1}$ имеет место интегральное равенство $[v, w]_{P}=\int_{P} w^{i} d y$.

В работе [14] было доказано неравенство (см. в [14] оценку (13)) для функций $v \in H^{1}(P)^{d}$

$$
\int_{P} v_{i} v_{i} d y \leqslant C_{8}\left(\int_{S} v_{n}^{2} d s+\int_{P} e_{i k, y}(v) e_{i k, y}(v) d y\right),
$$

где $v_{n}=v \cdot n, C_{8}=\operatorname{const}(P)$. Из $(26)$ и неравенства Корна для области $P$ (см. [4], [15]) следует, что $[v, v]^{1 / 2}$ есть норма в пространстве $V_{1}$. Стандартно, аналогично тому, как доказывалась теорема 1 , доказывается следуюшая лемма.

Лемма 2. Для задачи $\left(25_{i}\right), i=1, \ldots, d$, существует и единственно обобщенное решение $z^{i}(y)$, и для него выполнена оценка:

$$
\left[z^{i}, z^{i}\right]_{P} \leqslant C_{9}
$$

Из предложений 1 и 2 , а также леммы 2 следует существование для задачи Стокса $\left(25_{i}\right), i=1, \ldots, d$, не только обобщенной функции скорости $z^{i}(y)$, но и обобщенной функции давления $q^{i}(y) \in L^{2}(P) / \mathbb{R}$ такой, что

$$
\left|q^{i}\right|_{L^{2}(P) / \mathbb{R}} \leqslant C_{10}
$$

где $i=1, \ldots, d, L^{2}(P) / \mathbb{R}=\left\{v(y) \in L^{2}(P): \int_{P} v(y) d y=0\right\}$.

Так же, как в [2], [6], можно доказать, что если

$$
K_{i j}=\int_{P} z_{j}^{i}(y) d y, \quad i, j=1, \ldots, d
$$

где $z^{i}(y)=\left(z_{1}^{i}(y), \ldots, z_{d}^{i}(y)\right)$ - решение задачи $\left(25_{i}\right), i=1, \ldots, d$, то $\left\{K_{i j}\right\}_{i, j=1}^{d}$ есть симметрическая, положительно определенная матрица. Отсюда вытекает справедливость следуюшей леммы.

Лемма 3. Если $\left\{K_{i j}\right\}_{i, j=1}^{d}$ определена формулой (29), то для заданной функции $f \in L^{2}(\Omega)^{d}$ задача (1)-(3) относительно функции р имеет и притом единственное с точностью до аддитивной константы решение $p \in H^{1}(\Omega)$. 


\section{2. Продолжение решений.}

Лемма 4. Пусть $u^{\varepsilon}, p^{\varepsilon}$ - обобщеннъе решения задачи (7)-(10) для заданной функции $f \in L^{2}(\Omega)^{d}$. Тогда для әтих функций существуют продолжения (обозначаемые опять через $u^{\varepsilon}, p^{\varepsilon}$ ) в область $\Omega$, для которых справедливы равномернье по в при достаточно мальх $\varepsilon, \varepsilon \leqslant \varepsilon_{0}$, оченки

$$
\begin{gathered}
\varepsilon^{-1}\left|u^{\varepsilon}\right|_{H^{1}(\Omega)^{d}} \leqslant C_{11}, \quad \varepsilon^{-2}\left|u^{\varepsilon}\right|_{L^{2}(\Omega)^{d}} \leqslant C_{11}, \\
\left|\nabla p^{\varepsilon}\right|_{H^{-1}(\Omega)^{d}} \leqslant C_{12}, \quad\left|p^{\varepsilon}\right|_{L^{2}(\Omega) / \mathbb{R}} \leqslant C_{12} .
\end{gathered}
$$

ДокАЗАТЕЛЬСТво. Существование продолжения $u^{\varepsilon}$ на область $\Omega$, удовлетворяюшего оценке (30), следует из теорем продолжения для перфорированных областей, доказанных, например, в [4] или [5], при этом учитываются полученные уже оценки (22), (23), а также неравенства Корна для перфорированных областей $\Omega_{\varepsilon}$.

Сушествование продолжения $p^{\varepsilon}$ до функции из $L^{2}(\Omega) / \mathbb{R}$ с выполнением равномерных по $\varepsilon$ оценок (31) доказывается так же, как доказывалось аналогичное утверждение в приложении Тартара в [2]. При этом используется следуюшая лемма Тартара из [2].

Лемма 5. Существует линейный оператор $R_{\varepsilon}: H_{0}^{1}(\Omega)^{d} \rightarrow H_{0}^{1}\left(\Omega_{\varepsilon}\right)^{d}$ co следующими свойствами:

$$
\begin{gathered}
e c \Omega u w \in H_{0}^{1}\left(\Omega_{\varepsilon}\right)^{d}, \text { mo } R_{\varepsilon} w=w \\
e c \Omega u \operatorname{div} w=0, \text { mo } \operatorname{div} R_{\varepsilon} w=0 \\
\left|R_{\varepsilon} w\right|_{L^{2}\left(\Omega_{\varepsilon}\right)^{d}} \leqslant C_{13}\left(|w|_{L^{2}(\Omega)^{d}}+\varepsilon \sum_{i=1}^{d}\left|D_{i} w\right|_{L^{2}(\Omega)^{d}}\right) \\
\sum_{i=1}^{d}\left|D_{i} R_{\varepsilon} w\right|_{L^{2}\left(\Omega_{\varepsilon}\right)^{d}} \leqslant C_{13}\left(\varepsilon^{-1}|w|_{L^{2}(\Omega)^{d}}+\sum_{i=1}^{d}\left|D_{i} w\right|_{L^{2}(\Omega)^{d}}\right) .
\end{gathered}
$$

Следуя Тартару, определим продолжение $\nabla p^{\varepsilon}$ на область $\Omega$ функцией $F^{\varepsilon}$ такой, что

$$
\left\langle F^{\varepsilon}, w\right\rangle_{\Omega}=\left\langle\nabla p^{\varepsilon}, R_{\varepsilon} w\right\rangle_{\Omega_{\varepsilon}} \quad \forall w \in H_{0}^{1}(\Omega)^{d},
$$

где $R_{\varepsilon}$ - оператор из леммы 5, а $\langle\cdot, \cdot\rangle_{\Omega}$ обозначает отношение между функциями из пространств $H^{-1}(\Omega)^{d}$ и $H_{0}^{1}(\Omega)^{d}$, аналогично определяется $\langle\cdot, \cdot\rangle_{\Omega_{\varepsilon}}$.

Правую часть в $(36)$ перепишем в силу уравнения $(7)$, тогда

$$
\left\langle F^{\varepsilon}, w\right\rangle_{\Omega}=-\int_{\Omega_{\varepsilon}} D_{j}\left(u_{i}^{\varepsilon}\right) D_{j}\left(R_{\varepsilon} w\right) d x+\int_{\Omega_{\varepsilon}} f_{i}\left(R_{\varepsilon} w\right)_{i} d x
$$

так как $R_{\varepsilon} w \in H_{0}^{1}\left(\Omega_{\varepsilon}\right)^{d}$. Из $(32)$ следует, что $\left.F^{\varepsilon}\right|_{\Omega_{\varepsilon}}=\nabla p^{\varepsilon}$. Если $\operatorname{div} w=0$, то из (33) в силу (36) вытекает, что $\left\langle F^{\varepsilon}, w\right\rangle_{\Omega}=0$, и значит, в силу предложения $1 F^{\varepsilon}-$ градиент некоторой функции из $L^{2}(\Omega) / \mathbb{R}$. Таким образом, из предыдущих рассуждений видно, что $F^{\varepsilon}$ есть продолжение $\nabla p^{\varepsilon}$ на $\Omega$ и это продолжение само является градиентом некоторой функции $\tilde{p}^{\varepsilon}$ такой, что $\left.\tilde{p}^{\varepsilon}\right|_{\Omega_{\varepsilon}}=p^{\varepsilon}$. Далее продолжение $\tilde{p}^{\varepsilon}$ функции $p^{\varepsilon}$ на область $\Omega$ обозначается по-прежнему через $p^{\varepsilon}$. 
Оценим нормы продолжения $p^{\varepsilon}$. Из (37) на основании оценок $(34),(35)$, а также оценки (22) с учетом неравенства Корна при $\varepsilon \leqslant 1$ выводим

$$
\left|\left\langle\nabla p^{\varepsilon}, w\right\rangle_{\Omega}\right| \leqslant C_{14}\left(|w|_{L^{2}(\Omega)^{d}}+\varepsilon \sum_{i=1}^{d}\left|D_{i} w\right|_{L^{2}(\Omega)^{d}}\right) \leqslant|w|_{H^{1}(\Omega)^{d}}
$$

Из (38) и из определения $H^{-1}$-нормы вытекает первое неравенство из (31). Значит, в силу предложения $2 p^{\varepsilon} \in L^{2}(\Omega) / \mathbb{R}$, при этом

$$
\left|p^{\varepsilon}\right|_{L^{2}(\Omega) / \mathbb{R}} \leqslant C_{15}\left|\nabla p^{\varepsilon}\right|_{H^{-1}(\Omega)^{d}} \leqslant C_{12}
$$

Лемма 4 доказана.

Из оценок (31) следует, что по некоторой подпоследовательности $\left\{\varepsilon^{\prime}\right\} \subset\{\varepsilon\}$ (обозначаем ее далее по-прежнему через $\{\varepsilon\}$ ), стремящейся к нулю,

$$
\begin{gathered}
p^{\varepsilon} \rightarrow p \text { слабо в } L^{2}(\Omega) / \mathbb{R}, \\
\nabla p^{\varepsilon} \rightarrow \nabla p \text { слабо в } H^{-1}(\Omega)^{d} .
\end{gathered}
$$

Оказывается, сходимости (40), (41) можно усилить, используя специфику продолжения $p^{\varepsilon}$ на область $\Omega$, при котором выполняется первая из оценок (38).

ЛЕмма 6. Для введенного выше продолжсения на область $\Omega$ функиии $p^{\varepsilon}$, удовлетворяющего (40), (41), имеют место следующие сходимости:

$$
\begin{gathered}
\nabla p^{\varepsilon} \rightarrow \nabla p \text { сильно в } H^{-1}(\Omega)^{d} \\
p^{\varepsilon} \rightarrow p \text { сильно в } L^{2}(\Omega) / \mathbb{R}
\end{gathered}
$$

при $\varepsilon=\varepsilon^{\prime} \rightarrow 0$, где $\left\{\varepsilon^{\prime}\right\} \subset\{\varepsilon\}-$ некоторая подпоследовательность.

ДокАЗАТЕльство. 1) Прежде всего заметим справедливость следуюшего факта: если при $\varepsilon \rightarrow 0$

$$
w^{\varepsilon} \rightarrow w \text { слабо в } H_{0}^{1}(\Omega)^{d}
$$

и $\nabla p^{\varepsilon}$ удовлетворяет (41), то

$$
\left\langle\nabla p^{\varepsilon}, w^{\varepsilon}\right\rangle_{\Omega} \rightarrow\langle\nabla p, w\rangle_{\Omega}
$$

при $\varepsilon=\varepsilon^{\prime} \rightarrow 0$, где $\left\{\varepsilon^{\prime}\right\} \subset\{\varepsilon\}-$ некоторая подпоследовательность.

Действительно, в силу (38)

$$
\begin{aligned}
& \left|\left\langle\nabla p^{\varepsilon}, w\right\rangle_{\Omega}-\langle\nabla p, w\rangle_{\Omega}\right| \\
& \quad \leqslant\left|\left\langle\nabla p^{\varepsilon}, w^{\varepsilon}-w\right\rangle_{\Omega}\right|+\left|\left\langle\nabla p^{\varepsilon}-\nabla p, w\right\rangle_{\Omega}\right| \\
& \quad \leqslant C_{14}\left(\left|w^{\varepsilon}-w\right|_{L^{2}(\Omega)^{d}}+\varepsilon \sum_{i=1}^{d}\left|D_{i}\left(w-w^{\varepsilon}\right)\right|_{L^{2}(\Omega)^{d}}+\left|\left\langle\nabla p^{\varepsilon}-\nabla p, w\right\rangle_{\Omega}\right|\right) .
\end{aligned}
$$


В последней сумме из трех слагаемых последние два стремятся к нулю при $\varepsilon \rightarrow 0$ в силу (41), (44) и присутствия множителя $\varepsilon$. Первое же слагаемое этой суммы стремится к нулю по некоторой подпоследовательности $\left\{\varepsilon^{\prime}\right\} \subset\{\varepsilon\}$ в силу (44) и теоремы о компактном вложении $H_{0}^{1}(\Omega)$ в $L^{2}(\Omega)$. Таким образом, сходимость (45) доказана.

2) Пусть $g^{\varepsilon}=\nabla\left(p^{\varepsilon}-p\right)$ и $v^{\varepsilon}, v$ - решения краевой задачи

$$
-\Delta w=h \quad \text { в } \Omega, \quad w=0 \text { на } \partial \Omega,
$$

из пространства $H_{0}^{1}(\Omega)^{d}$, если в качестве $h$ взять, соответственно, $\nabla p^{\varepsilon}$ или $\nabla p$. Тогда

$$
\left|g^{\varepsilon}\right|_{H^{-1}(\Omega)^{d}}^{2}=\left\langle(-\Delta)^{-1} g^{\varepsilon}, g^{\varepsilon}\right\rangle_{\Omega}=\left\langle v^{\varepsilon}, g^{\varepsilon}\right\rangle_{\Omega}-\left\langle v, g^{\varepsilon}\right\rangle_{\Omega} .
$$

И в силу сходимости (41) имеем $\left\langle v, g^{\varepsilon}\right\rangle_{\Omega} \rightarrow 0$. Кроме того, из (41) следует, что $v^{\varepsilon} \rightarrow v$ слабо в $H_{0}^{1}(\Omega)^{d}$. Поэтому $\left\langle v^{\varepsilon}, g^{\varepsilon}\right\rangle_{\Omega}=\left\langle v^{\varepsilon}, \nabla p^{\varepsilon}\right\rangle_{\Omega}-\left\langle v^{\varepsilon}, \nabla p\right\rangle_{\Omega} \rightarrow 0$ при $\varepsilon=\varepsilon^{\prime} \rightarrow 0$ на основании п. 1). И значит, $\left|g^{\varepsilon}\right|_{H^{-1}(\Omega)^{d}} \rightarrow 0$ при $\varepsilon=\varepsilon^{\prime} \rightarrow 0$, что эквивалентно сходимости (42). Сходимость (43) вытекает автоматически из (42), если воспользоваться первой оценкой из (39). Лемма 6 доказана.

4.3. Закон Дарси и его обоснование. В этом пункте будет сформулирована и доказана основная теорема настоящей работы, дающая обоснование закона Дарси для задачи Стокса с условием неполного прилипания на границе.

Tеорема 2. Пусть $f \in L^{2}(\Omega)^{d} u u^{\varepsilon}, p^{\varepsilon}-$ продолжения решений задачи (7)-(10) на область $\Omega$, введеннье в $n .4 .2$. Пусть $K=\left\{K_{i j}\right\}_{i, j=1}^{d}$ тензор, определенный формулой (29). Тогда

$$
\varepsilon^{-2} u^{\varepsilon} \rightarrow \text { ислабо в } L^{2}(\Omega)^{d}, \quad p^{\varepsilon} \rightarrow \text { сильно в } L^{2}(\Omega) / \mathbb{R}
$$

при $\varepsilon \rightarrow 0$, где $u=K(f-\nabla p)$, при әтом функиия $р$ является решением задачи (1)-(3).

ДокАЗАТЕЛЬство. 1) Введем вспомогательные функции

$$
z_{\varepsilon}^{i}(x) \equiv z^{i}\left(\frac{x}{\varepsilon}\right), \quad q_{\varepsilon}^{i} \equiv q^{i}\left(\frac{x}{\varepsilon}\right), \quad i=1, \ldots, d,
$$

где пара функций $\left(z^{i}(y), q^{i}(y)\right)$ определяется как решение вспомогательной задачи $\left(25_{i}\right), i=1, \ldots, d$, на ячейке $P$. Эти вспомогательные функции удовлетворяют следуюшим соотношениям в области $\omega_{\varepsilon}$ (см. определение в п. 2.3 ) и на ее границе:

$$
\begin{gathered}
\Delta_{x} z_{\varepsilon}^{i}-\varepsilon^{-1} \nabla_{x} q_{\varepsilon}^{i}+\varepsilon^{-2} e_{i}=0 \text { в } \omega_{\varepsilon}, \\
\operatorname{div} z_{\varepsilon}^{i}=0 \text { в } \omega_{\varepsilon} \\
z_{\varepsilon}^{i} \cdot n=0 \text { на } \partial \omega_{\varepsilon} \\
T_{\tau, x}\left(z_{\varepsilon}^{i}(x), \varepsilon^{-1} q_{\varepsilon}^{i}(x)\right)=0 \text { на } \partial \omega_{\varepsilon} .
\end{gathered}
$$

Справедливость (51) вытекает из того, что

$$
T_{i j, x}\left(z_{\varepsilon}^{i}(x), \varepsilon^{-1} q_{\varepsilon}^{i}(x)\right)=\varepsilon^{-1} T_{i j, y}\left(z^{i}(y), q^{i}(y)\right),
$$


где нижний индекс $x$ или $y$ при компоненте тензора указывает, по какой переменной $(x$ или $y)$ проводится дифференцирование, когда определяется эта компонента.

Из (50), (51), используя разложение (12), можно вывести

$$
\int_{\partial \omega_{\varepsilon}} T_{i j, x}\left(z_{\varepsilon}^{i}(x), \varepsilon^{-1} q_{\varepsilon}^{i}(x)\right) n_{j} \varphi_{k} d s=0 .
$$

Кроме того, для функций $z_{\varepsilon}^{i}(x), q_{\varepsilon}^{i}(x)$ справедливы оценки, вытекающие из ограниченности области $\Omega$ и соответствующих оценок для функций $z^{i}(y), q^{i}(y)$,

$$
\begin{gathered}
\left|z_{\varepsilon}^{i}(x)\right|_{L^{2}(\Omega)^{d}} \leqslant C, \quad\left|q_{\varepsilon}^{i}(x)\right|_{L^{2}(\Omega)} \leqslant C, \\
\sum_{j=1}^{d}\left|D_{j} z_{\varepsilon}^{i}(x)\right|_{L^{2}(\Omega)^{d}} \leqslant \varepsilon^{-1} C, \quad \sum_{j=1}^{d}\left|D_{j} q_{\varepsilon}^{i}(x)\right|_{L^{2}(\Omega)} \leqslant \varepsilon^{-1} C,
\end{gathered}
$$

где константа $C$ не зависит от $\varepsilon$ и от $i$.

2) В дальнейшем будет использована оценка из работы [16] (см. лемму 2 в [16]), согласно которой в векторном случае справедливо следующее

$$
\begin{array}{r}
\left|\varepsilon \int_{S_{\varepsilon}} v \cdot w d s-\Lambda \int_{\Omega_{\varepsilon}} v \cdot w d x\right| \\
\leqslant \varepsilon C_{15} \sum_{i, i=1}^{d}\left(\left|D_{i} v\right|_{L^{2}\left(\Omega_{\varepsilon}\right)^{d}}|w|_{L^{2}\left(\Omega_{\varepsilon}\right)^{d}}+\left|D_{i} w\right|_{L^{2}\left(\Omega_{\varepsilon}\right)^{d}}|v|_{L^{2}\left(\Omega_{\varepsilon}\right)^{d}}\right. \\
\left.+\varepsilon\left|D_{i} v\right|_{L^{2}\left(\Omega_{\varepsilon}\right)^{d}}\left|D_{j} w\right|_{L^{2}\left(\Omega_{\varepsilon}\right)^{d}}\right)
\end{array}
$$

где $\Lambda=(\operatorname{mes} S)(\operatorname{mes} P)^{-1}$, mes $M-r$-мера для $r$-мерного множества $M, v, w \in$ $H^{1}\left(\Omega_{\varepsilon}, S_{\varepsilon}\right)^{d}$.

3 ) Далее фиксируем одно из возможных значений $i, i=1, \ldots, d$. Возьмем $\Phi(x)=$ $\psi(x) z_{\varepsilon}^{i}(x)$, где $\psi(x)$ - произвольная функция из $D(\Omega), z_{\varepsilon}^{i}$ - функция, определенная в п. 1) доказательства теоремы, продолженная нулем на $\Omega \backslash \Omega_{\varepsilon}$. Далее также рассматриваем решение $p^{\varepsilon}(x)$ задачи $(7)-(10)$, продолженное на всю область $\Omega$ так, как указано в п. 4.2.

Рассмотрим выражение $I(\varepsilon) \equiv-\int_{\Omega} \nabla p^{\varepsilon} \cdot \Phi d x$. Преобразуем его двумя способами: сначала интегрированием по частям, а затем - в силу уравнения (7), и далее перейдем к пределу при $\varepsilon \rightarrow 0$.

При первом способе преобразования $I(\varepsilon)$ введем под знак градиента константу $C_{\varepsilon}$ такую, что $\int_{\Omega}\left(p^{\varepsilon}-p+C_{\varepsilon}\right) d x=0$, где $p$ - предельная функция из (43). От этого внесения, очевидно, значение $I(\varepsilon)$ не изменится. А в силу $(49)$ и $(50)$ после интегрирования по частям в $I(\varepsilon)$ получаем представление для $I(\varepsilon)$

$$
I(\varepsilon)=\int_{\Omega_{\varepsilon}}\left(p^{\varepsilon}+C_{\varepsilon}\right) \frac{\partial \psi}{\partial x_{j}} z_{\varepsilon j}^{i} d x,
$$

при этом использовано условие (49). И поскольку (см., например, [2; гл. V, лемма 4.1]) $z_{\varepsilon}^{i}(x) \rightarrow \int_{P} z_{j}^{i}(y) d y \equiv K_{i j}$ слабо в $L^{2}(\Omega)$ при $\varepsilon \rightarrow 0$, то в силу леммы 6 при 
$\varepsilon=\varepsilon^{\prime}$ (где $\left\{\varepsilon^{\prime}\right\}$ - некоторая подпоследовательность $\{\varepsilon\}$ ) имеем $p^{\varepsilon}-p+C_{\varepsilon} \rightarrow 0$ в

$L^{2}(\Omega)$ и, значит, при $\varepsilon=\varepsilon^{\prime} \rightarrow 0$

$$
I(\varepsilon) \rightarrow \int_{\Omega} p \frac{\partial \psi}{\partial x_{j}} K_{i j} d x .
$$

Преобразуем $I(\varepsilon)$ вторым способом в силу уравнения (7)

$$
I(\varepsilon)=-\int_{\Omega_{\varepsilon}} f \cdot \Phi d x-\nu \int_{\Omega_{\varepsilon}} \Delta u^{\varepsilon} \cdot \Phi d x \equiv I_{1}(\varepsilon)+I_{2}(\varepsilon) .
$$

Легко установить следуюшую сходимость: при $\varepsilon \rightarrow 0$

$$
I_{1}(\varepsilon) \equiv-\int_{\Omega_{\varepsilon}} f \cdot \Phi d x=-\int_{\Omega}\left(f \cdot z_{\varepsilon}^{i}(x)\right) \psi d x \rightarrow \int_{\Omega} K_{i j} f_{j} \psi d x .
$$

Поэтому осталось перейти к пределу при $\varepsilon \rightarrow 0$ в слагаемом $I_{2}(\varepsilon)$ суммы $(56)$. Для этого преобразуем $I_{2}(\varepsilon)$, интегрируя его по частям и используя соленоидальность вектора $u^{\varepsilon}=\left(u_{1}^{\varepsilon}, \ldots, u_{d}^{\varepsilon}\right)$ :

$$
\begin{aligned}
I_{2}(\varepsilon) & \equiv-\nu \int_{\Omega_{\varepsilon}} \Delta u^{\varepsilon} \cdot \Phi d x=-\nu \int_{\Omega_{\varepsilon}} \frac{\partial}{\partial x_{k}}\left(\frac{\partial u_{j}^{\varepsilon}}{\partial x_{k}}+\frac{\partial u_{k}^{\varepsilon}}{\partial x_{j}}\right) \Phi_{j} d x \\
& =\nu \int_{\Omega_{\varepsilon}} e_{j k}\left(u^{\varepsilon}\right) \frac{\partial \Phi_{j}}{\partial x_{k}} d x-\nu \int_{S_{\varepsilon}} e_{j k}\left(u^{\varepsilon}\right) n_{k} \Phi_{j} d s .
\end{aligned}
$$

Далее через $r_{m}(\varepsilon), m=0,1, \ldots$, будем обозначать слагаемые, которые стремятся к нулю при $\varepsilon \rightarrow 0$. Тогда так как $\Phi \cdot n=0$ на $S_{\varepsilon}$, что следует из краевого условия (50) для функции $z_{\varepsilon}^{i}(x)$, участвуюшей в определении функции $\Phi$, то

$$
\int_{S_{\varepsilon}} p^{\varepsilon} \delta_{j k} n_{k} \Phi_{j} d s=\int_{S_{\varepsilon}} p^{\varepsilon}(\Phi \cdot n) d s .
$$

Поэтому ввиду формулы (4) и краевого условия (17) для функции $u^{\varepsilon}$ на границе $S_{\varepsilon}$ имеем представление для второго слагаемого правой части (58)

$$
\begin{aligned}
\nu \int_{S_{\varepsilon}} e_{j k}\left(u^{\varepsilon}\right) n_{k} \Phi_{j} d s & =\int_{S_{\varepsilon}} T_{j k}\left(u^{\varepsilon}, p^{\varepsilon}\right) n_{k} \Phi_{j} d x \\
& =-\lambda \int_{S_{\varepsilon}} u^{\varepsilon} \cdot \Phi d s=-\lambda \int_{S_{\varepsilon}}\left(u^{\varepsilon} \cdot z_{\varepsilon}^{i}\right) \psi d s=r_{0}(\varepsilon),
\end{aligned}
$$

где на заключительном этапе использованы оценки (54) и (22), (23).

Далее, преобразуем первое слагаемое правой части (58), используя симметричность тензора $e_{j k}\left(u^{\varepsilon}\right)$, а также интегрирование по частям:

$$
\begin{aligned}
\nu \int_{\Omega_{\varepsilon}} e_{j k}\left(u^{\varepsilon}\right) \frac{\partial \Phi_{j}}{\partial x_{k}} d x & =\frac{\nu}{2} \int_{\Omega_{\varepsilon}} e_{j k}\left(u^{\varepsilon}\right) e_{j k}(\Phi) d x=\nu \int_{\Omega_{\varepsilon}} \frac{\partial u_{j}^{\varepsilon}}{\partial x_{k}} e_{j k}(\Phi) d x \\
& =-\nu \int_{\Omega_{\varepsilon}} u_{j}^{\varepsilon} \frac{\partial}{\partial x_{k}} e_{j k}(\Phi) d x+\nu \int_{S_{\varepsilon}} e_{j k}(\Phi) n_{k} u_{j}^{\varepsilon} d s .
\end{aligned}
$$


При этом

$$
\int_{S_{\varepsilon}} e_{j k}(\Phi) n_{k} u_{j}^{\varepsilon} d s=\int_{S_{\varepsilon}} e_{j k}\left(z_{\varepsilon}^{i}\right) \psi n_{k} u_{j}^{\varepsilon} d s+r_{1}(\varepsilon)
$$

где $r_{1}(\varepsilon) \rightarrow 0$ при $\varepsilon \rightarrow 0$. Это справедливо, так как

$$
r_{1}(\varepsilon) \equiv \int_{S_{\varepsilon}}\left(z_{\varepsilon j}^{i} \frac{\partial \psi}{\partial x_{k}}+z_{\varepsilon k}^{i} \frac{\partial \psi}{\partial x_{j}}\right) n_{k} u_{j}^{\varepsilon} d s=\int_{S_{\varepsilon}} \frac{\partial \psi}{\partial n}\left(z_{\varepsilon}^{i} \cdot u^{\varepsilon}\right) d s
$$

в силу того, что $z_{\varepsilon}^{i} \cdot n=0$ на $S_{\varepsilon}$ и правую часть последнего интегрального равенства можно оценить, учитывая оценку (54), а также неравенства (53), (22), (23), которьм удовлетворяют функции $z_{\varepsilon}^{i}, u^{\varepsilon}$.

Заметим, что

$$
\int_{S_{\varepsilon}} \delta_{j k} q_{\varepsilon}^{i}(x) n_{k} u_{j}^{\varepsilon} d s=\int_{S_{\varepsilon}} q_{\varepsilon}^{i}(x)\left(u^{\varepsilon} \cdot n\right) d s=0
$$

в силу краевого условия для функции $u^{\varepsilon}$ на $S_{\varepsilon}$. Поэтому формулу (61) можно переписать в следующем виде:

$$
\int_{S_{\varepsilon}} e_{j k}(\Phi) n_{k} u_{j}^{\varepsilon} d s=\nu^{-1} \int_{S_{\varepsilon}} T_{j k, x}\left(z_{\varepsilon}^{i}, \varepsilon^{-1} q_{\varepsilon}^{i}(x) \psi n_{k} u_{j}^{\varepsilon} d s+r_{1}(\varepsilon)=r_{1}(\varepsilon)\right.
$$

при этом использовано равенство (52).

В итоге из представления $I_{2}(\varepsilon)$ в виде суммы $(58)$ в силу полученных формул (59), (60), (62) вытекает сначала равенство

$$
I_{2}(\varepsilon)=\nu \int_{\Omega_{\varepsilon}} u_{j}^{\varepsilon} \frac{\partial}{\partial x_{k}} e_{j k}(\Phi) d x+r_{1}(\varepsilon)+r_{0}(\varepsilon)
$$

а вслед за ним в силу соленоидальности $z_{\varepsilon}^{i}(x)$ и оценок $(53),(22),(23)$ - равенство

$$
I_{2}(\varepsilon)=\nu \int_{\Omega_{\varepsilon}}\left(u^{\varepsilon} \cdot \Delta z_{\varepsilon}^{i}\right) \psi d x+r_{2}(\varepsilon)
$$

Далее, принимая во внимание равенство (48), соленоидальность векторного поля $u^{\varepsilon}$, а также краевое условие (10) и оценки (53) и (22), выводим

$I_{2}(\varepsilon)=\int_{\Omega_{\varepsilon}} \varepsilon^{-2}\left(u^{\varepsilon} \cdot e^{i}\right) \psi d x-\varepsilon^{-1} \int_{\Omega_{\varepsilon}}\left(\nabla q_{\varepsilon}^{i} \cdot u^{\varepsilon}\right) \psi d x+r_{2}(\varepsilon)=\varepsilon^{-2} \int_{\Omega} u_{i}^{\varepsilon} \psi d x+r_{3}(\varepsilon)$.

Поэтому, используя слабую компактность $\left\{\varepsilon^{-2} u^{\varepsilon}(x)\right\}$ в $L^{2}(\Omega)$ в силу оценки (30), получаем сходимость по некоторой подпоследовательности $\left\{\varepsilon^{\prime}\right\} \subset\{\varepsilon\}, \varepsilon^{\prime} \rightarrow 0$,

$$
I_{2}(\varepsilon) \rightarrow \int_{\Omega} u_{i} \psi d x
$$


Таким образом, ввиду представления (56) из доказанных сходимостей (55), (57) и (64) вытекает

$$
\int_{\Omega} p K_{i j} \frac{\partial \psi}{\partial x_{j}} d x=-\int_{\Omega} K_{i j} f_{j} \psi d x+\int_{\Omega} u_{i} \psi d x
$$

или

$$
\int_{\Omega} K_{i j}\left(f_{j}-\frac{\partial p}{\partial x_{j}}\right) \psi d x=\int_{\Omega} u_{i} \psi d x
$$

что в силу произвольности выбора функции $\psi$, а также параметра $i$ означает

$$
u_{i}=K_{i j}\left(f_{j}-\frac{\partial p}{\partial x_{j}}\right), \quad i=1, \ldots, d
$$

И значит, связь между предельными функциями из сходимостей (46) установлена. Кроме того, предельное векторное поле $u$ наследует соленоидальность векторного поля $u^{\varepsilon}$

$$
\int_{\Omega} u \nabla \varphi d x=0
$$

для произвольной функции $\varphi \in D(\Omega)$. Равенства (65), (66) означают, что предельные функции $u, p$ удовлетворяют краевой задаче (1)-(3). Заметим, что $p$ как элемент $L^{2}(\Omega) / \mathbb{R}$ и $u$ как элемент $L^{2}(\Omega)^{d}$ определяются из этой задачи однозначно по заданной функции $f$. Поэтому, хотя пара $u, p$ была получена как предельная для сходимостей (46) лишь по некоторой подпоследовательности $\left\{\varepsilon^{\prime}\right\} \subset\{\varepsilon\}$ такой, что $\varepsilon^{\prime} \rightarrow 0$, можно утверждать, что вся последовательность $\left\{\varepsilon^{-2} u^{\varepsilon}\right\}$ и вся последовательность $\left\{p^{\varepsilon}\right\}$ при $\varepsilon \rightarrow 0$ имеют сходимость (46). Значит, утверждение теоремы 2 доказано.

\section{Список литературы}

1. Полубаринова-Кочина П. Я. Теория движения грунтовых вод. М.: Наука, 1977.

2. Санчес-Паленсия Е. Неоднородные среды и теория колебаний. М.: Мир, 1984.

3. Бахвалов Н. С., Панасенко Г. П. Осреднение процессов в периодических средах. М.: Наука, 1984.

4. Олейник О.А., Иосифьян Г. А., Шамаев А. С. Математические задачи теории сильно неоднородных сред. М.: Изд-во МГУ, 1990.

5. Жиков В.В., Козлов С. М., Олейник О. А. Усреднение диффференциальных операторов. М.: Наука, 1993.

6. ЖКиков В. В. Об усреднении уравнений Стокса в перфорированной области // Докл. PAH. 1994. T. 334. № 2. C. 144-147.

7. Beliaev A. Yu., Kozlov S. M. Darcy Equation for Random Porous Media // CPAM. 1996. V. 49. № 1. P. 1-35.

8. Беляев А.Ю., Эффендиев Я.Р. Усреднение системы уравнений Стокса // Матем. заметки. 1996. Т. 59. № 4. С. 504-520.

9. Слезкин Н. А. Динамика вязкой несжимаемой жидкости. М.: Гостехиздат, 1954.

10. Петров Н. П. Трение в машинах и влияние на него смазьвающей жидкости // Гидродинамическая теория смазки. М.: ГТТИ, 1934. С. 11-247.

11. Ладыженская О.А. Математические вопросы вязкой несжимаемой жидкости. М.: Наука, 1970.

12. Темам Р. Уравнения Навье-Стокса. Теория и численньй анализ. М.: Мир, 1981. 
13. Сандраков Г. В. Осреднение нестационарной системы Стокса в перфорированной области // Изв. РАН. Сер. матем. 1997. Т. 61. №1. С. 113-140.

14. Пастухова C.E. О характере затухания перемещений в упругом периодически перфорированном теле с заданной деформацией внешней границы при упругих связях на границе полостей // Дифференц. уравнения. 1996. Т. 32. № 10. С. 1401-1409.

15. Дюво Г., Лионс Ж.-Л. Неравенства в механике и физике. М.: Наука, 1980.

16. Пастухова C. E. Метод компенсированной компактности Тартара в усреднении спектра смешанной задачи для эллиптического уравнения в перфорированной области с третьим краевьм условием // Матем. сб. 1995. Т. 186. № 5. С. 127-144.

Московский государственньй институт

радиотехники, электроники и автоматики

(технический университет)
Поступила в редакцию

05.11.1997 